\title{
Quantitative Comparative Proteomics Reveals Candidate Biomarkers for the Early Prediction of Gestational Diabetes Mellitus: A Preliminary Study
}

\author{
DANAI MAVRELI ${ }^{1,2}$, NIKOLAS EVANGELINAKIS $^{2}$, NIKOLAS PAPANTONIOU $^{1}$ and AGGELIKI KOLIALEXI ${ }^{1,2}$ \\ ${ }^{1} 3^{\text {rd }}$ Department of Obstetrics and Gynecology, \\ National and Kapodistrian University of Athens Medical School, Athens, Greece \\ ${ }^{2}$ Department of Medical Genetics, National and Kapodistrian University of Athens Medical School, Athens, Greece
}

\begin{abstract}
Aim: To identify differentially expressed proteins (DEPS) in $1^{\text {st }}$ trimester maternal plasma between pregnant women at risk for gestational diabetes mellitus (GDM) and uncomplicated controls. Materials and Methods: Firsttrimester plasma from five women who developed GDM and five from non-diabetic ones were analyzed using isobaric tag for relative and absolute quantitation - labeled proteomics. Enzyme-linked immunosorbent assay was further applied in an independent cohort of 25 GDM cases and 25 controls for verification. Results: Prenylcysteine oxidase 1 (PCYOX1), beta-ala-his dipeptidase (CNDP1), extracellular matrix protein 1 (ECM1), basement membrane-specific heparan sulfate proteoglycan core protein (HSPG2), thrombospondin4 (TSP-4) demonstrated significant differences in expression between the two groups $(p<0.05)$. DEPs are mainly associated with complement and coagulation cascades. Conclusion: The reported plasma proteomic changes represent potential biomarkers for the early identification of women at risk for GDM. Future studies using larger and more diverse cohorts are necessary to assess the clinical utility of these findings.
\end{abstract}

Gestational diabetes mellitus (GDM) is a common metabolic pregnancy-related complication defined as carbohydrate

This article is freely accessible online.

Correspondence to: Aggeliki Kolialexi, Department of Medical Genetics, National and Kapodistrian University of Athens, School of Medicine, Children's Hospital "Aghia Sophia", Choremeio Research Institute (2nd Floor), Thivon \& Levadeias, Athens 115 27, Greece. Tel: +30 2107467463, Fax: +30 2107795553, e-mail: akolial@med.uoa.gr

Key Words: Biomarkers, GDM, gestational diabetes mellitus, mass spectrometry, TMT isobaric labeling, nano-LC-MS/MS, prenatal screening, pregnancy complications. intolerance resulting in hyperglycemia with onset or first diagnosis during pregnancy (1). Depending on the population studied and the criteria employed for screening, the prevalence ranges between 1-14\% with growing incidence, which is associated with the increased prevalence of obesity among women of reproductive age $(2,3)$. Although the pathogenetic mechanism leading to GDM is poorly understood, the cause of the condition is linked to insulin resistance and insulin deficiency.

GDM is associated with various adverse consequences for both, the mother and their offspring, including preeclampsia, cesarean delivery, preterm birth, macrosomia, fetal malformations, hyperbilirubinemia and neonatal metabolic disorders as well as an exceptionally high risk for developing cardiovascular disease and type 2 diabetes mellitus (T2DM) after the index pregnancy $(4,5)$.

Currently, in clinical practice GDM is diagnosed using the oral glucose tolerance test (OGTT) at 24-28 weeks of pregnancy (1). Even though this test is minimally invasive, it requires a hospital visit and multiple blood draws. Furthermore, it is offered at 24-28 weeks of pregnancy, when sufficient fetal adipose tissue has developed (6). Earlier detection, however, and more accurate diagnostic methods will be highly beneficial for patients as it will lead to increased diagnosis rates, improved perinatal outcomes through timely interventions and prevent maternal and offspring risk for future metabolic disease.

Mass spectrometry-based comparative proteomic profiling of maternal plasma could provide a deeper insight in the pathogenesis of the disease. Through identification of GDMrelated non-invasive predictive and diagnostic biomarkers it can broaden the horizons on disease screening, diagnosis and treatment (7). The underlying rationale for such studies is that proteins secreted into maternal circulation reflect physiological or disease states and alterations in expression levels are indicative of pregnancies at risk. However, due to 
in vivo $34: 517-525(2020)$

Table I. Maternal and pregnancy characteristics of women participating in the study.

\begin{tabular}{|c|c|c|c|c|c|c|}
\hline & $\begin{array}{l}\text { GDM } \\
(\mathrm{n}=5)\end{array}$ & $\begin{array}{l}\text { Controls } \\
(\mathrm{n}=5)\end{array}$ & $p$-Value & $\begin{array}{l}\text { GDM } \\
(\mathrm{n}=25)\end{array}$ & $\begin{array}{l}\text { Controls } \\
(\mathrm{n}=25)\end{array}$ & $p$-Value \\
\hline Maternal age & $\begin{array}{c}26.58 \\
(23.4-30.3)\end{array}$ & $\begin{array}{c}26.2 \\
(25.4-27.9)\end{array}$ & NS* & $\begin{array}{c}31.81 \\
(21.2-39.5)\end{array}$ & $\begin{array}{c}33.19 \\
(26.7-41.2)\end{array}$ & NS* \\
\hline $\begin{array}{l}\text { Pre-pregnancy BMI } \\
\left(\mathrm{Kg} / \mathrm{m}^{2}\right)\end{array}$ & $\begin{array}{c}23.1 \\
(21.5-23.5)\end{array}$ & $\begin{array}{c}22.8 \\
(21.3-23.2)\end{array}$ & NS* & $\begin{array}{c}27.10 \\
(25.5-29.1)\end{array}$ & $\begin{array}{c}26.60 \\
(24.10-30.8)\end{array}$ & NS* \\
\hline Parity & 0 & 0 & - & $1(0-3)$ & $1(0-3)$ & NS* \\
\hline OGTT-fast (mmol/l) & $\begin{array}{c}5.6 \\
(3.92-4.81)\end{array}$ & $\begin{array}{c}4.32 \\
(4.10-4.55)\end{array}$ & $<0.001$ & $\begin{array}{c}5.9 \\
(5.2-6.1)\end{array}$ & $\begin{array}{c}4.22 \\
(3.8-4.6)\end{array}$ & $<0.001$ \\
\hline OGTT $-1 \mathrm{~h}(\mathrm{mmol} / \mathrm{l})$ & $\begin{array}{c}11.4 \\
(10.9-11.9)\end{array}$ & $\begin{array}{c}9.1 \\
(8.6-9.3)\end{array}$ & $<0.001$ & $\begin{array}{c}11.7 \\
(10.9-12.1)\end{array}$ & $\begin{array}{c}9.2 \\
(8.2-1.7)\end{array}$ & $<0.001$ \\
\hline OGTT- $2 \mathrm{~h}(\mathrm{mmol} / \mathrm{l})$ & $\begin{array}{c}8.4 \\
(8.3-9.1)\end{array}$ & $\begin{array}{c}7.9 \\
(7.7-8.0)\end{array}$ & $<0.001$ & $\begin{array}{c}8.9 \\
(8.6-9.2)\end{array}$ & $\begin{array}{c}7.9 \\
(7.4-7.8)\end{array}$ & $<0.001$ \\
\hline Gestational age at delivery & $\begin{array}{c}38.8 \\
(37.9-9.1)\end{array}$ & $\begin{array}{c}38.4 \\
(38.1-38.9)\end{array}$ & NS* & $\begin{array}{c}38.9 \\
(38.2-39.4)\end{array}$ & $\begin{array}{c}39.1 \\
(38.7-40.2)\end{array}$ & 0.35 \\
\hline \multicolumn{7}{|l|}{ Mode of delivery } \\
\hline $\begin{array}{l}\text { Induced delivery }(\mathrm{n}) \\
\text { Cesarean section }(\mathrm{n})\end{array}$ & $\begin{array}{l}3 \\
2\end{array}$ & $\begin{array}{l}1 \\
4\end{array}$ & - & $\begin{array}{c}21 \\
4\end{array}$ & $\begin{array}{c}19 \\
6\end{array}$ & - \\
\hline Birth weight & $\begin{array}{c}3,372 \\
(2,980-3,550)\end{array}$ & $\begin{array}{c}3,345 \\
(2,950-3,500)\end{array}$ & NS* & $\begin{array}{c}3,390 \\
(3,050-3,570)\end{array}$ & $\begin{array}{c}3,480 \\
(3,320-3,610)\end{array}$ & NS* \\
\hline \multicolumn{7}{|l|}{ Fetal gender } \\
\hline Boys (n) & 3 & 4 & - & 10 & 13 & - \\
\hline Girls (n) & 2 & 1 & & 15 & 12 & \\
\hline
\end{tabular}

NS*: $p<0.05$.

the complexity and the high dynamic range of the plasma proteome, identification of plasma biomarkers remains challenging and state-of-the-art quantitative approaches allowing precise assessment of low-abundance proteins in mixed samples are required.

The present study aims to identify proteins with significantly altered expression in the $1^{\text {st }}$ trimester maternal plasma, in women subsequently diagnosed with GDM and their matched controls via employment of tandem mass tags (TMT) isobaric labeling followed by nano-liquid chromatography-tandem mass spectrometry (nLC-MS/MS). These proteins represent candidate biomarkers for prediction and diagnosis of GDM at an earlier stage and may provide important clues for the mechanisms of this pregnancy related complication.

\section{Materials and Methods}

Study participants. Peripheral blood samples for this case-control study were retrospectively selected for analysis from the maternity plasma bio-bank at the $3^{\text {rd }}$ department of Obstetrics and Gynecology of Athens University School of Medicine. Patients were recruited between 2015 and 2017. Blood samples were collected from women undergoing $1^{\text {st }}$ trimester (11-13 weeks of gestation) prenatal screening for fetal aneuploidies into EDTA- tubes and centrifuged at $2500 \times \mathrm{g}$ for $10 \mathrm{~min}$ at $4^{\circ} \mathrm{C}$. Plasma was aliquoted in sterile vacutainers supplemented with protease inhibitor and stored at $-80^{\circ} \mathrm{C}$ for subsequent analysis.
Women's demographic and clinical data including results of the OGTT test, which is routinely offered to all pregnant women at our hospital, were recorded in the hospital records and reviewed by the obstetricians involved in the project for patient selection. A total of 60 samples were retrieved for analysis. Maternal clinical characteristics and pregnancy outcomes are shown in Table I.

During the first phase of the study, the discovery step, plasma samples from 5 women subsequently diagnosed with GDM and 5 non-diabetic participants (controls) were analyzed using TMT isobaric labeling coupled with n-LC-MS/MS in order to identify proteins showing significant differences in expression levels between GDM and control samples. GDM cases and controls were matched for maternal age, parity and pre-pregnancy body mass index (BMI).

At the second phase, the differential expression of selected proteins, identified during the discovery step was verified by performing enzyme-linked immunosorbent assay (ELISA) in an independent cohort of $25 \mathrm{GDM}$ cases and an equal number of nonGDM controls randomly selected.

All women included in the study had a singleton pregnancy, were free from any medical disorders or pregnancy complications and delivered phenotypically normal neonates at $>37$ weeks' gestation. Pregnancies ending in termination or miscarriage were excluded as were smokers, women who underwent assisted reproductive treatment and those with a previous delivery of a large for gestational age neonate. None of the samples were previously thawed and refrozen.

In accordance with the guidelines of the American College of Obstetricians and Gynecologists women were considered to have GDM if venous plasma glucose levels reached or exceeded one or more of the following criteria: a fasting morning plasma glucose of 
$92 \mathrm{mg} / \mathrm{dl}(5.1 \mathrm{mM}) ;$ a $1 \mathrm{~h}$ post-load $75 \mathrm{~g}$ oral glucose of $180 \mathrm{mg} / \mathrm{dl}$ $(10 \mathrm{mM}) ;$ a $2 \mathrm{~h}$ post-load glucose of $153 \mathrm{mg} / \mathrm{dl}(8.2 \mathrm{mM})(8)$. Control participants had a negative OGTT result and did not developed GDM during pregnancy.

The Scientific and Ethics Committee at the "Attikon" University Hospital Greece approved the study protocol (approval reference number: 27-268 ex 14/15). All women gave written informed consent to participate in the study, which was performed in accordance with the relevant guidelines and regulations.

Sample preparation and mass spectrometry analysis.

Depletion of high-abundance plasma proteins. To increase the accuracy of proteomic analysis, human plasma was depleted of abundant proteins (albumin and antibody components) using the Pierce $^{\mathrm{TM}}$ Top 2 Abundant Protein Depletion Spin Columns (Thermo Fisher Scientific ${ }^{\mathrm{TM}}$, Waltham, MA, USA) as per the manufacturer's specifications. The total protein concentration was determined using the Pierce ${ }^{\mathrm{TM}}$ BCA Protein Assay Kit (Thermo Fisher Scientific ${ }^{\mathrm{TM}}$, Waltham, MA, USA).

Protein tryptic digestion and TMT reagent labeling. For digestion, 40 $\mu \mathrm{g}$ of depleted plasma proteins from each sample were reduced by $10 \mathrm{mM}$ Tris-(2-carboxy-ethyl) phosphine (TCEP) at $56^{\circ} \mathrm{C}$ for 1 $\mathrm{h}$, alkylated by addition of $20 \mathrm{mM}$ iodoacetamide for $1 \mathrm{~h}$ at room temperature in the dark and treated by trypsin protease (Promega, Madison, WI, USA) at a ratio of $1: 100$ at $37^{\circ} \mathrm{C}$ over-night to generate proteolytic peptides (9). After trypsin digestion, peptides were lyophilized to near dryness. Peptide-containing solutions from each sample (GDM or control) were separately labeled using TMT10plex ${ }^{\mathrm{TM}}$ Isobaric Label Reagent Set (Thermo Fisher Scientific ${ }^{\mathrm{TM}}$, Waltham, MA, USA), according to the manufacturer's instructions. Labeled peptides were mixed together at equal amount before further analysis.

High-performance liquid chromatography (HPLC) fractionation. To reduce sample complexity the labeled peptides were loaded onto a C18 column $(10 \mathrm{~mm} \times 250 \mathrm{~mm}, 5 \mu \mathrm{m})$ (Thermo Fisher Scientific ${ }^{\mathrm{TM}}$, Waltham, MA, USA) for peptide fractionation. The mobile phase was a mixture of $0.1 \%$ formic acid in water (A) and $0.1 \%$ formic acid in acetonitrile (B) with a liquid chromatography (LC) linear gradient of $8-32 \%$ buffer B over $60 \mathrm{~min}$. Each mixed sample was fractionated into 6 sections for subsequent mass spectrometry analysis.

nanoLC-MS/MS analysis. nano-LC-MS/MS was carried out on an Orbitrap Q Exactive ${ }^{\mathrm{TM}}$ HF mass spectrometer with an electro-spray ionization nano-spray source coupled on-line to a Ultimate-3000 system (Thermo Fisher Scientific ${ }^{\mathrm{TM}}$, Waltham, MA, USA).

Briefly, $5 \mu$ of labeled peptide mixtures were injected onto a trapping column (PepMap C18, 100A, $100 \mu \mathrm{m} \times 2 \mathrm{~cm}, 5 \mu \mathrm{m}$ ) and washed with buffer A ( $0.1 \%$ formic acid in water). Following valve switching, peptides were separated on an analytical column (PepMap C18, 100A, $75 \mu \mathrm{m} \times 50 \mathrm{~cm}, 2 \mu \mathrm{m}$ ) at a constant flow of $250 \mathrm{nl} / \mathrm{min}$ and eluted with a linear gradient from 5 to $7 \%$ buffer B ( $0.1 \%$ formic acid in acetonitrile) in $2 \mathrm{~min}$, from $7 \%$ to $20 \%$ buffer B in $80 \mathrm{~min}$, from $20 \%$ to $40 \%$ buffer B in $35 \mathrm{~min}$, then from $40 \%$ to $90 \%$ buffer $\mathrm{B}$ in $4 \mathrm{~min}$. The instrument was operated in datadependent acquisition mode (IDA). Data were collected in a positive ion mode over a broad mass to charge $(\mathrm{m} / \mathrm{z})$ precursor ion selection scan range of $\mathrm{m} / \mathrm{z} 300-1650$. The 15 most intense ions were isolated for fragmentation by collision-induced dissociation at $40 \%$ normalized collision energy (NCE) (10).
Data analysis. The raw data were analyzed using the Maxquant (version 1.5.1.0) software to identify proteins and peptides and searched against the human proteome of reference found at UNIPROT Knowledgebase v.2.16 (http://www.uniprot.org) (11). The parameters were set as follows: the protein modifications were carbamidomethylation (C) (fixed), oxidation (M) (variable); the enzyme specificity was set to trypsin; the maximum missed cleavages were set to 2 ; the precursor ion mass tolerance was set to $10 \mathrm{ppm}$ and MS/MS tolerance was $0.6 \mathrm{Da}$. Only peptides/proteins identified at a False Detection Rate (FDR) $<1 \%$ were accepted. For protein abundance ratios, we used fold change $>1.5$ or $<0.83$ as the threshold.

Gene ontology and pathway analysis. Gene ontology (GO) annotation of the differentially expressed proteins was performed in three aspects: biological process (BP), molecular function (MF) and cellular component (CC) via employment of the Webgestalt webtool (12). KEGG pathway enrichment analysis was conducted using the Database for Annotation, Visualization and Integrated Discovery (DAVID) online tools with the cut-off criterion of $p<0.05$ (13).

ELISA analysis. ELISA analysis was performed to verify the quantitative proteomics results. First trimester maternal plasma samples from an independent cohort of pregnant women (25 with GDM cases and 25 controls) were analyzed using commercially available kits for thombospondin-4 (TSP-4) (\#OKAG00254) and beta-ala-his dipeptidase (CNDP1) (\#OKEH00851) (Aviva Systems Biology, Corp., San Diego, CA, USA) according to the manufacturer's instructions.

Statistical analysis. Statistical tests were performed using MATLAB ${ }^{\circledR}$ (The Mathworks, Inc, Natick, MA). Differences in clinical characteristics between the two groups were assessed using ANOVA for independent samples or the Mann-Whitney $U$-test for continuous variables.

Receiver operating characteristic (ROC) curves were carried out to generate the diagnostic potential of differentially expressed proteins with $95 \%$ standard error (SE) and $95 \%$ confidence intervals (CI). Significant differences were defined as two-tailed $p$ value $<0.05$.

\section{Results}

NanoLC-MS/MS comparative proteomic analysis. On the basis of data acquisition, a total of 238 DEPs demonstrating a $>1.5$ and $<0.8$ were identified: 198 $(83,2 \%)$ up-regulated and $40(16.8 \%)$ down-regulated. Among them, five proteins namely prenylcysteine oxidase 1 [PCYOX1 (Q9UHG3)], beta-ala-his dipeptidase [CNDP1 (Q96KN2)], extracellular matrix protein 1 [ECM1 (Q16610)], basement membrane-specific heparan sulfate proteoglycan core protein [HSPG2 (P98160)], thrombospondin-4 [TSP-4 (P49744)] demonstrated a statistically significant altered expression between the two groups $(p<0.05)$. TSP-4 was the most up-regulated protein, and its levels were increased by 3.4-fold in GDM cases as compared to normoglycemic ones. No protein with significant down-regulation was detected. 
Bioinformatics analysis of the differentially expressed proteins. By applying the gene ontology (GO) classification of the abundant molecules identified by n-LC-MS/MS, according to their biological processes, the following categories emerged: biological regulation $(n=215)$, metabolic process $(n=208)$, response to stimulus $(n=205)$, localization $(n=168)$, multicellular organismal processing $(n=167)$, cellular component organization $(n=116)$, development $(n=115)$, cell communication $(n=102)$ and other classes such as multi-organism process, cell proliferation, growth and reproduction in lower numbers (Figure 1A).

Clustering of identified proteins as differentially expressed in GDM cases, under the term "Cellular Components", predicted that the majority are extracellular $(n=226)$, followed by vesicles $(n=190)$ and proteins localized in the endomembrane system $(n=127)$ (Figure 1B). Other significantly enriched categories include the membrane-enclosed lumen, membranes, protein containing complexes and the extracellular matrix as well as proteins residing in the nucleus, cytosol and organelle compartmentalization such as mitochondria, endoplasmatic reticulum, Golgi apparatus and ribosomes.

Associations of the DEPs using their "Molecular function" as criterion are illustrated in Figure 1C. The top-5 overrepresented terms include protein binding activity $(\mathrm{n}=181)$, ion binding $(n=115)$, hydrolases $(n=69)$, enzyme regulators $(n=50)$ and lipid binding $(n=38)$.

Through application of the Kyoto Encyclopedia Genes and Genomes (KEGG) bioinformatics software, proteins with altered expression levels in the first trimester maternal plasma of GDM cases were found mainly engaged in the complement and coagulation cascades, staphylococcus aureus infection, prion diseases and cholesterol metabolism (Figure 2).

Verification of protein expression by ELISA. Consistent with the n-LC-MS/MS data, ELISA analysis verified the overexpression of TSP-4 and CNDP1 in the first trimester maternal plasma in women who subsequently developed GDM (Figure 3). Both proteins were examined for their performance in differentiating between GDM and control samples. The area under the curve (AUC) obtained for TSP4 was 0.94 and for CND1 0.98 at $p<0.001$ (Figure 4).

\section{Discussion}

The present study focused on the detection of differential protein expression patterns between GDM and normoglycemic pregnant women, to determine whether specific $1^{\text {st }}$ trimester maternal plasma proteins might represent candidate early predictors for the complication. The comparative proteomic analysis was carried out using nLC-MS/MS. In order to increase the coverage of protein identification and the confidence in the data generated, proteins were labeled with TMT isobaric labeling reagents.
To the best of our knowledge, only two studies have previously applied TMT isobaric labeling combined with nLC-MS/MS on screening and identifying novel candidate plasma biomarkers for GDM, using well-defined clinical samples obtained early during gestation. Zhao et al., analyzed plasma collected from women in the early $2 \mathrm{nd}$ trimester of gestation (12-16 weeks) and reported 31 proteins as differentially expressed in the GDM group mainly involved in blood coagulation, inflammation, immune response and complement activation (14). Ravnsborg et al. using serum from obese women at 11-13 weeks of pregnancy reported afamin, serum amyloid P-component and vitronectin as potential biomarkers for the complication (15).

In the present study, a total of 238 DEPs were identified. Out of those, 198 were up-regulated and 40 down-regulated. KEGG pathway enrichment analysis revealed that several of these proteins are implicated in the coagulation and complement pathway. The up-regulation of coagulation factors II (prothrombin) (P00734), V (P12259), IX (P00740), $\mathrm{X}$ (P00742) and XII (P00748) observed in the GDM group as compared to uncomplicated pregnancies suggests an exaggeration of the existing hyper-coagulation state associated with pregnancy (16). The list was accompanied by the altered expression of all three fibrinogen- polypeptide chains $[\alpha$ (P02671), $\beta$ (P02675) and $\gamma$ (P02679)] probably due to a common thrombocytosis state in the GDM patients $(17,18)$. Complement C3 (P01024), an important player in the activation of the complement system, in both, classical and alternative pathways, was found moderately overexpressed in the present study emphasizing the essential role of the immune system activation in the pathogenesis of GDM. Conflicting evidence, however, exists regarding C3 levels in GDM cases. Increased levels of C3 have been associated with diabetes and insulin resistance (19-21). Shen et al., however, reported decreased complement C3 concentration in GDM samples (6). Another significant finding of the present study is the moderate up-regulation of vitronectin (P04004), which has been previously identified as an independent candidate biomarker for GDM. Additionally, over-expression of the complement factor H (P08603) was observed, which has been previously associated with various metabolic disorders and obesity $(6,22)$.

A major finding of the present study is the significantly altered expression $(p<0.05)$ of CNDP1, ECM1, HSPG2, PCYOX1 and TSP-4, which is novel and has not been previously associated with the complication.

CNPD1 is synthesized in the brain and secreted into the systematic circulation (23). CNDP1 hydrolyzes L-carnosine and is essential for glucose metabolism and the transportation of activated fatty acids across the mitochondrial membrane $(24,25)$. A genetic polymorphism of CNDP1 has been associated with decreased serum levels of L-carnosine and diabetic nephropathy in patients with 
(A) Bar chart of Biological Process categories

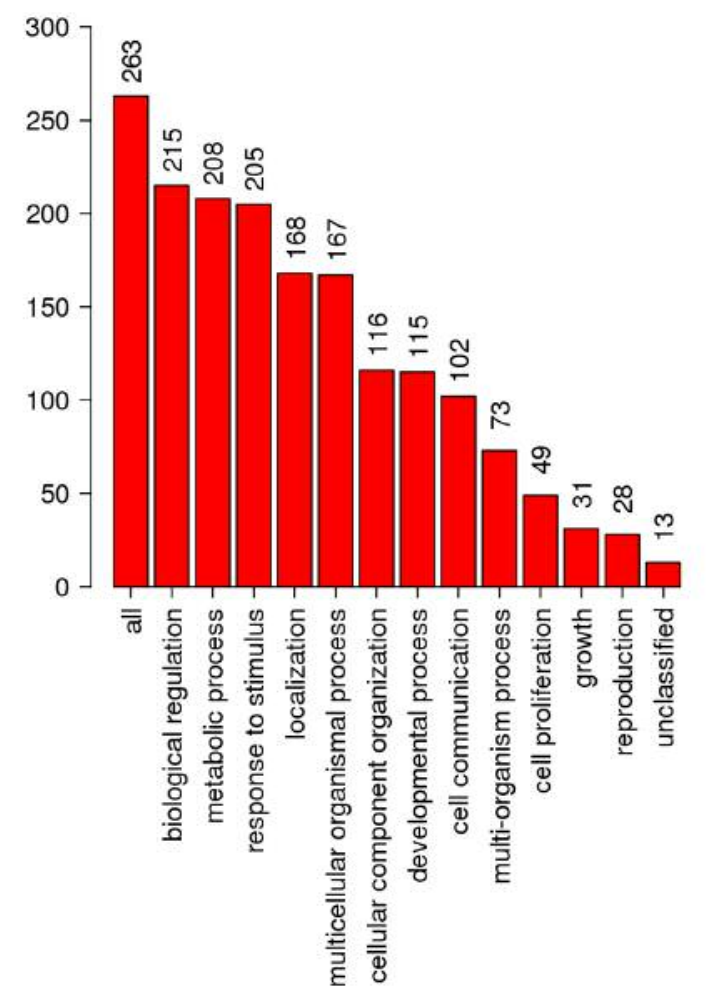

(B) Bar chart of Cellular Component categories

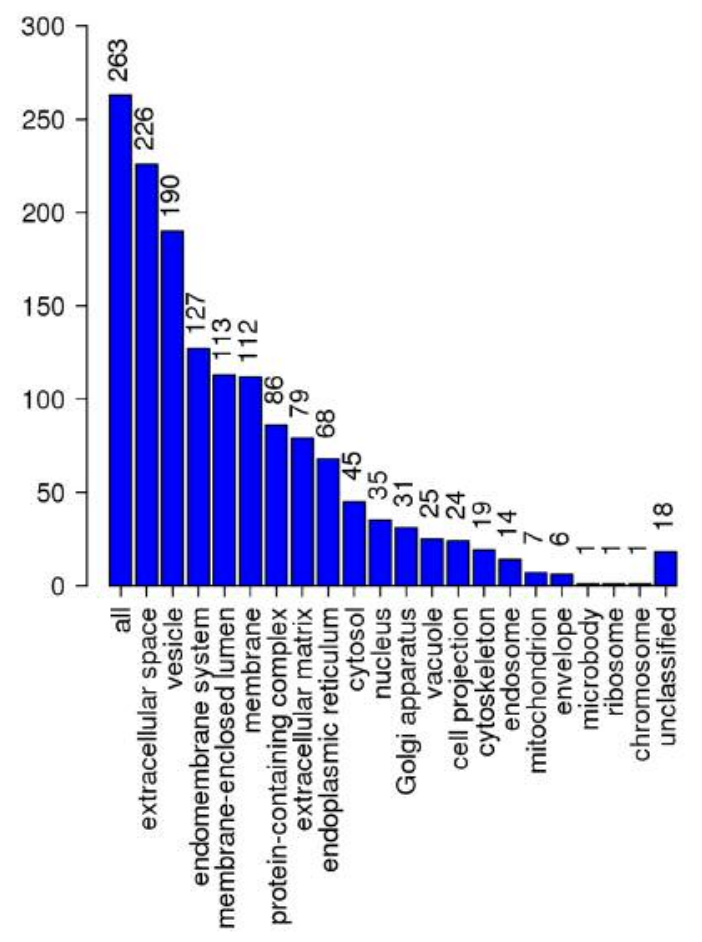

(C) Bar chart of Molecular Function categories

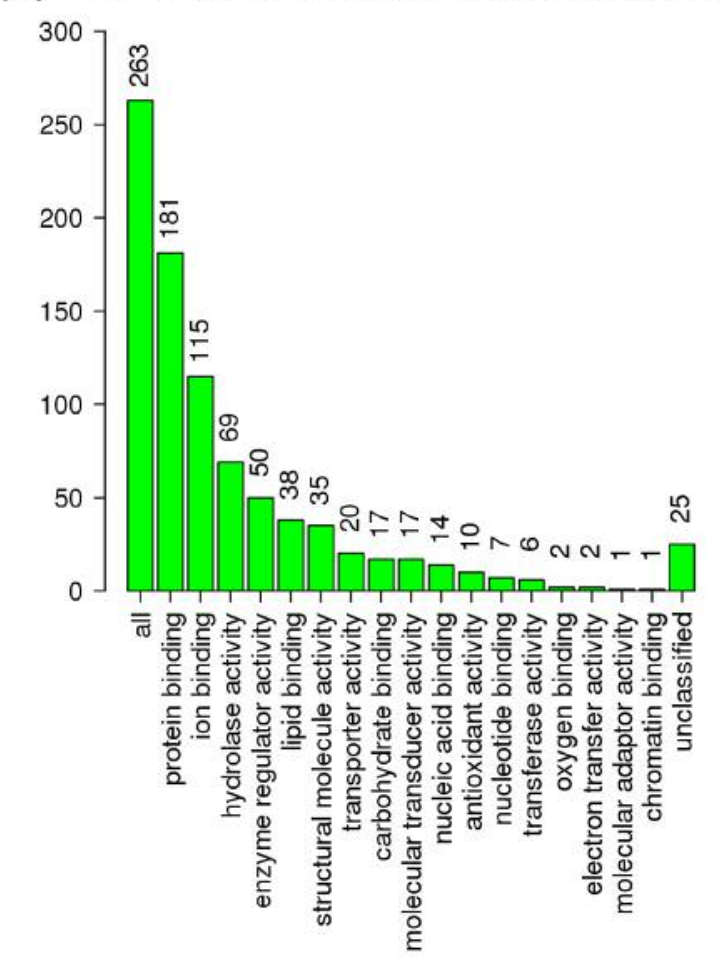

Figure 1. Gene ontology $(G O)$ categories of the abundant proteins in GDM cases according to (A) biological process, (B) cellular component and (C) molecular function. 


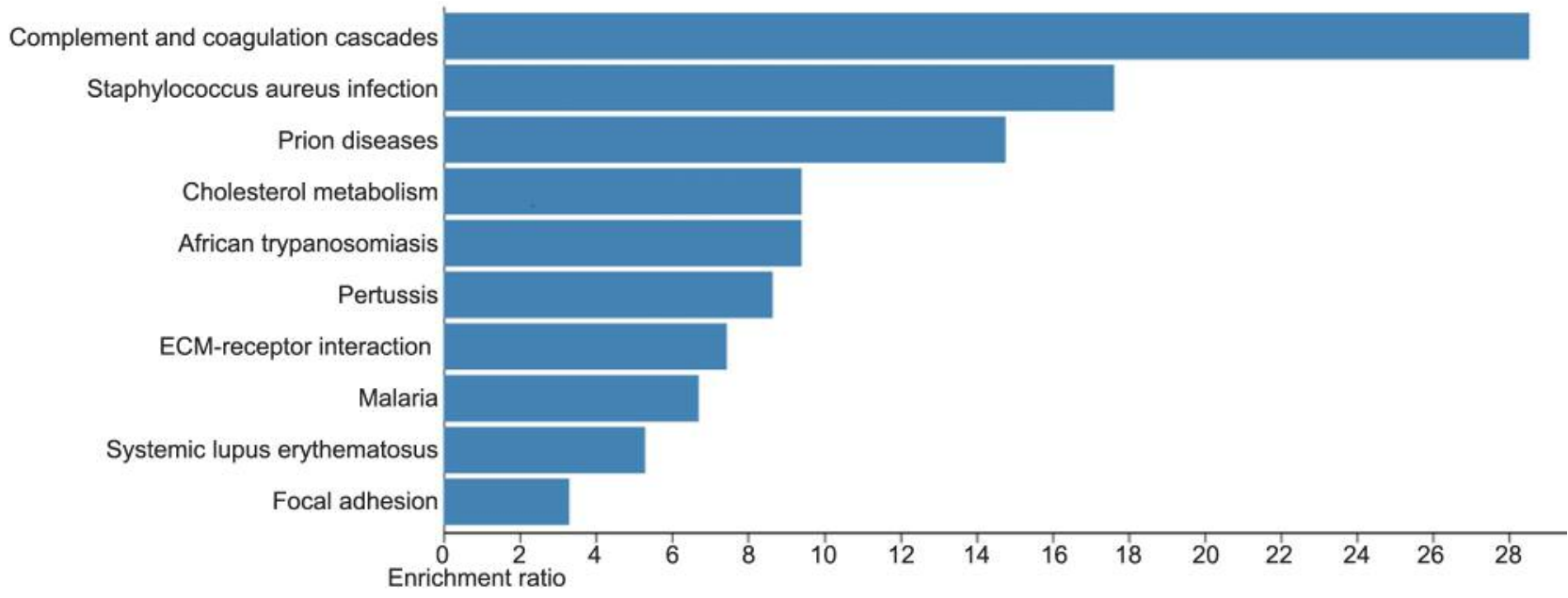

Figure 2. Kyoto Encyclopedia of Genes and Genomes pathways enriched with annotated differentially expressed miRNAs in the first trimester maternal plasma in GDM cases.

T1DM and T2DM suggesting a protective role of $\mathrm{L}$ carnosine for late complications of diabetic disease (26). Sauerhofer et al., in a mouse model mimicking T2DM pathology, demonstrated that lowered endogenous Lcarnosine serum levels were associated with glycosuria, higher fasting plasma glucose levels and glycosylated haemoglobin A1c levels as well as decreased body weight throughout life (25). In contrast, supplementation of Lcarnosine resulted in a milder diabetes manifestation indicating an association between CNDP1 expression levels and the pathogenesis of T2DM.

PCYOX1 is broadly expressed in various embryonic and adult tissues, predominantly the liver (27). It interacts with various proteins and plays an important role in the respiratory chain, cell death, cell signaling, movement and transport, metabolism and protein degradation. Increased PCYOX1 mRNA expression levels have been detected in the visceral adipose tissue of mice that were receiving a high fat diet whereas decreased PCYOX1 expression was reported in a group of patients under coptis chinensis administration, a common treatment for diabetes in China $(27,28)$. These observations combined with the findings from the present study suggest that PCYOX1 should be further investigated in relation to the pathogenesis of diabetes.

According to our findings increased ECM1 $11^{\text {st }}$ trimester plasma expression levels may have a crucial role in the initiation of GDM. ECM1 is a main component of the extracellular matrix involved in bone formation, angiogenesis in cancer and endothelial cell proliferation (2931). Oliva et al., identified altered expression levels of ECM1 in the adipose tissue of pregnant women who subsequently developed GDM (29). They hypothesized that this increase in ECM1 protein expression levels results in higher rigidity in the adipose tissue, promoting fibrosis. This hypothesis is supported by a recent study by Fan et al., who demonstrated that high expression levels of ECM1 inhibit transforming growth factor beta (TGFB) activation to prevent liver fibrosis (32). Lee et al. investigated the functional role of ECM1 and concluded that it interacts with the epidermal growth factor (EGF) domain of various proteins, including HSPG2, and induce heparin-binding growth factor responses (33). HSPG2 also known as perlecan, is a heparan sulfate proteoglycan involved in catabolism of lipids and glucose lipoprotein metabolism, obesity and the onset of metabolic syndrome (34).

In our initial proteomic study TSP-4 was significantly upregulated in the GDM group, showing a $>3$-fold change, the largest difference in a protein's expression level observed in the present study. TSP-4 is a member of the thrombospondin family, implicated in the regulation of endothelial cell and smooth muscle cell proliferation, fibrosis, cardiac hypertrophy, inflammation, the production of heparin sulfate core proteins and the remodeling of the myocardium in rats (35). In contrast to the rest molecules of the thrombospondin family, TSP-4 promotes angiogenesis $(36,37)$. Recently, Zierfuss et al. found increased levels of TSP-4 in T2DM patients with peripheral arterial disease suggesting that microvascular angiogenesis promoted in diabetes is associated with the overexpression of TSP-4 (38).

The strength of the present study lies in the use of a sensitive and reproducible mass spectrometry-based technique followed by ELISA confirmatory experiments to analyze plasma samples obtained from well-characterized GDM cases. Notably, several of the proteins identified in the 

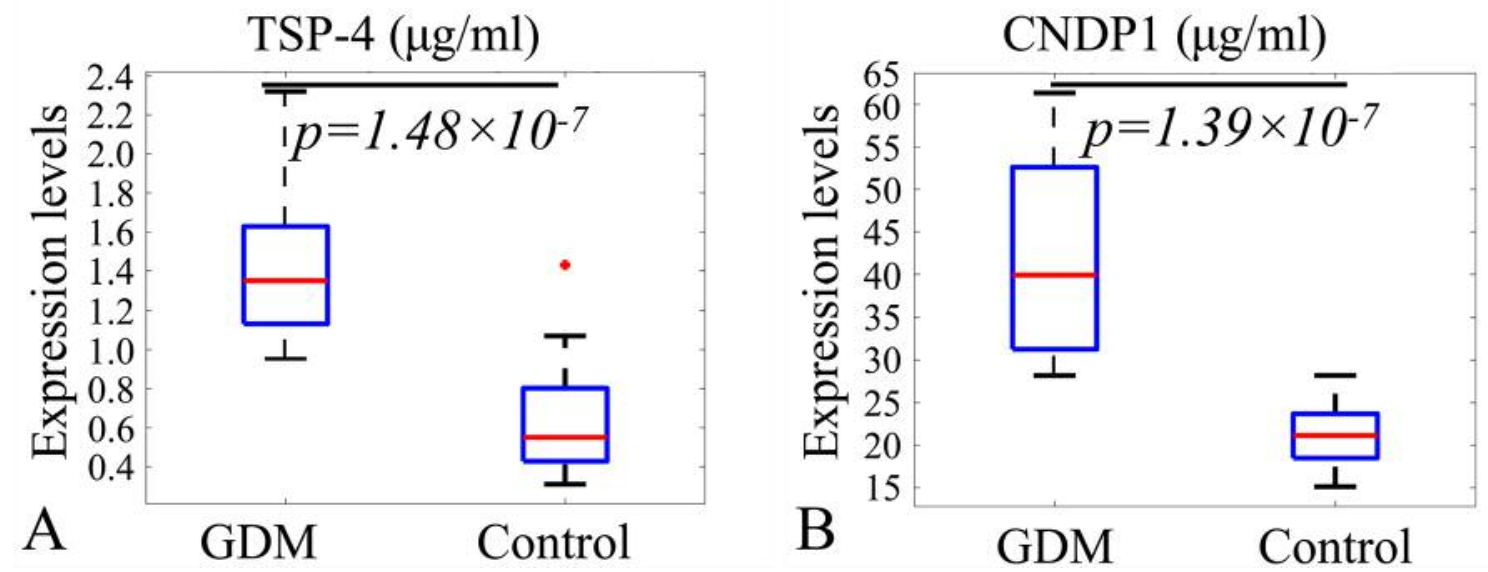

Figure 3. Box plot of TSP- 4 (A) and CNDP1 (B) results of GDM and the control groups (p-value $<0.001)$.
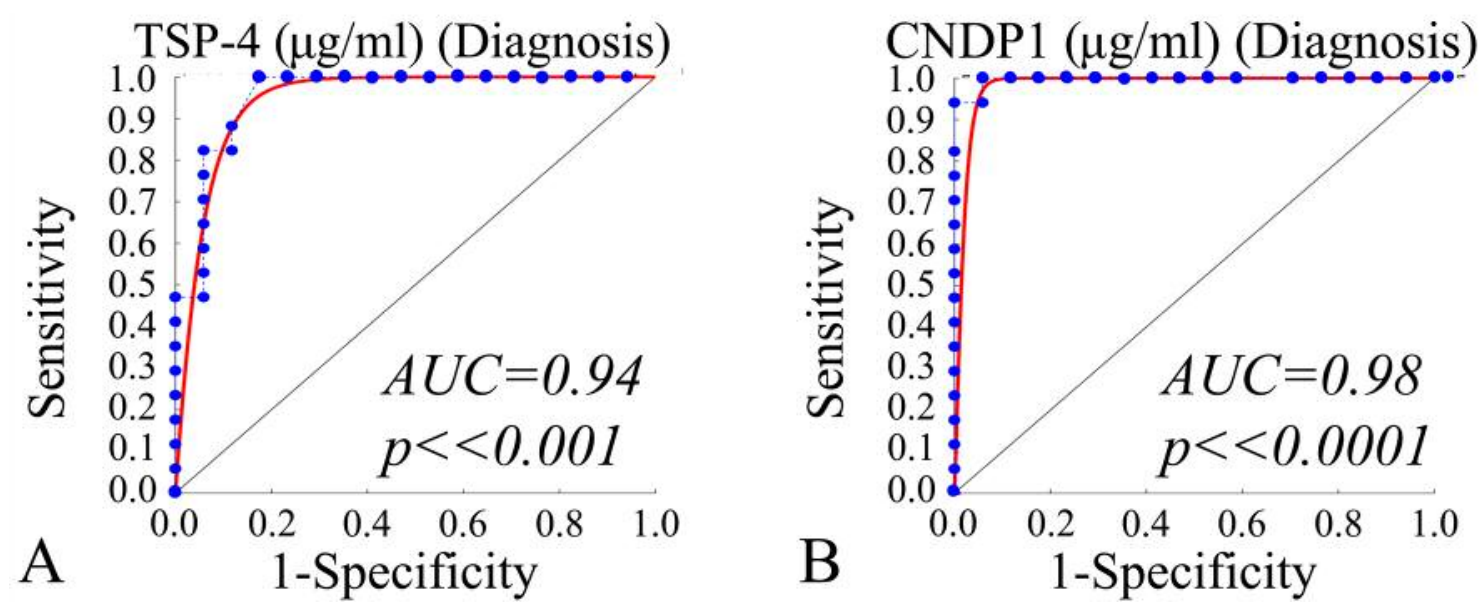

Figure 4. Receiver operating characteristic (ROC) curves showing the performance of TSP-4 (A) and CNDP1 (B) as individual diagnostic biomarkers.

present study have been previously reported in association with the later development of GDM including sex hormonebinding globulin (SHBG), adiponectin and C-reactive protein (CRP) as well as afamin, serum amyloid P-component and vitronectin reported as candidate biomarkers $(15,39,40)$. Even though, their altered expression was not statistically significant, their detection further supports a potential role as GDM predictors and adds strength to the findings of the present study. We acknowledge, however, that the limited number of cases and the weakness of the case-control study design applied may have led to an overestimation. For this reason, further studies using a larger population representative of various ethnic groups are required to validate the data obtained and determine their performance in early predicting GDM.

Using proteomic analyses we identified over-expression of CNDP1, ECM1, HSPG2, PCYOX1 and TSP-4 in the $1^{\text {st }}$ trimester maternal plasma of women who were subsequently diagnosed with GDM followed by confirmatory ELISA. These proteins show great potential to be used as early predictive biomarkers for prenatal screening as well as guiding the development of new therapeutic interventions. Our research, however, is preliminary and future studies is using a larger and more diverse cohort are necessary to validate these initial findings, assess their clinical utility and uncover their role in the pathogenesis of GDM. 


\section{Funding}

The research was co-funded by the European Union and Greek national funds through the Operational Program "Human Resources Development, Education and Lifelong Learning" (project code: MIS 5007192).

\section{Conflicts of Interest}

The Authors declare that there are no conflicts of interest.

\section{Authors' Contributions}

DM performed all experiments, participated in the interpretation of data analyses and drafted the manuscript. NE performed patients' diagnosis and treatment, sample and data collection and assisted in all experiments' performance. NP participated in the design and coordination of the study and supervised the study. AK conceived and designed the study, and drafted the manuscript. All Authors approved the final manuscript.

\section{Acknowledgements}

The Authors acknowledge the support of Dr. G. Lambrou in statistical and bioinformatic analyses.

\section{References}

1 American Diabetes Association: Gestational diabetes mellitus. Diabetes Care 26 Suppl 1: S103-105, 2003. PMID: 12502631. DOI: $10.2337 /$ diacare.26.2007.s103

2 Dabelea D, Snell-Bergeon JK, Hartsfield CL, Bischoff KJ, Hamman RF, McDuffie RS and Kaiser Permanente of Colorado GDMSP: Increasing prevalence of gestational diabetes mellitus (GDM) over time and by birth cohort: Kaiser permanente of Colorado GDM screening program. Diabetes Care 28(3): 579584, 2005. PMID: 15735191. DOI: 10.2337/diacare.28.3.579

3 Getahun D, Nath C, Ananth CV, Chavez MR and Smulian JC: Gestational diabetes in the united states: Temporal trends 1989 through 2004. Am J Obstet Gynecol 198(5): 525 e521-525, 2008. PMID: 18279822. DOI: 10.1016/j.ajog.2007.11.017

4 Reece EA: The fetal and maternal consequences of gestational diabetes mellitus. J Matern Fetal Neonatal Med 23(3): 199-203, 2010. PMID: 20121460. DOI: 10.3109/14767050903550659

5 McKenzie-Sampson S, Paradis G, Healy-Profitos J, St-Pierre F and Auger N: Gestational diabetes and risk of cardiovascular disease up to 25 years after pregnancy: A retrospective cohort study. Acta Diabetol 55(4): 315-322, 2018. PMID: 29327149. DOI: $10.1007 / \mathrm{s} 00592-017-1099-2$

6 Shen L, Zhao D, Chen Y, Zhang K, Chen X, Lin J, Li C, Iqbal J, Zhao Y, Liang Y, Wei Y and Feng C: Comparative proteomics analysis of serum proteins in gestational diabetes during early and middle stages of pregnancy. Proteomics Clin Appl 13(5): e1800060, 2019. PMID: 31162828. DOI: 10.1002/prca.201800060

7 Kolialexi A, Mavreli D and Papantoniou N: Proteomics for early prenatal screening of pregnancy complications: A 2017 perspective. Expert Rev Proteomics 14(2): 113-115, 2017. PMID: 28002974. DOI: 10.1080/14789450.2017.1275574
8 Committee on Practice B-O: Acog practice bulletin no. 190: Gestational diabetes mellitus. Obstet Gynecol 131(2): e49-e64, 2018. PMID: 29370047. DOI: 10.1097/AOG.0000000000002501

9 Tsangaris GT, Papathanasiou C, Adamopoulos PG, Scorilas A, Vorgias CE, Prodromou N, Stathopoulou FT, Stravopodis DJ and Anagnostopoulos AK: Pediatric ependymoma: A proteomics perspective. Cancer Genomics Proteomics 14(2): 127-136, 2017. PMID: 28387652. DOI: 10.21873/cgp.20025

10 Jedrychowski MP, Huttlin EL, Haas W, Sowa ME, Rad R and Gygi SP: Evaluation of hcd- and cid-type fragmentation within their respective detection platforms for murine phosphoproteomics. Mol Cell Proteomics 10(12): M111 009910, 2011. PMID: 21917720. DOI: $10.1074 / \mathrm{mcp} . M 111.009910$

11 Kwon OK, Ha YS, Lee JN, Kim S, Lee H, Chun SY, Kwon TG and Lee S: Comparative proteome profiling and mutant protein identification in metastatic prostate cancer cells by quantitative mass spectrometry-based proteogenomics. Cancer Genomics Proteomics 16(4): 273-286, 2019. PMID: 31243108. DOI: 10.21873/cgp.20132

12 Kaur S, Larramendy ML, Gentile M, Svarvar C, KoivistoKorander R, Vauhkonen H, Scheinin I, Leminen A, Butzow R, Bohling $\mathrm{T}$ and Knuutila $\mathrm{S}$ : New insights into the cellular pathways affected in primary uterine leiomyosarcoma. Cancer Genomics Proteomics 3(6): 347-354, 2006. PMID: 31394667.

13 Dennis G, Jr., Sherman BT, Hosack DA, Yang J, Gao W, Lane HC and Lempicki RA: David: Database for annotation, visualization, and integrated discovery. Genome Biol 4(5): P3, 2003. PMID: 12734009.

14 Zhao D, Shen L, Wei Y, Xie J, Chen S, Liang Y, Chen Y and Wu $\mathrm{H}$ : Identification of candidate biomarkers for the prediction of gestational diabetes mellitus in the early stages of pregnancy using itraq quantitative proteomics. Proteomics Clin Appl 11(78), 2017. PMID: 28220636. DOI: 10.1002/prca.201600152

15 Ravnsborg T, Svaneklink S, Andersen LLT, Larsen MR, Jensen DM and Overgaard M: First-trimester proteomic profiling identifies novel predictors of gestational diabetes mellitus. PLoS One 14(3): e0214457, 2019. PMID: 30917176. DOI: 10.1371/journal.pone. 0214457

16 Thornton P and Douglas J: Coagulation in pregnancy. Best Pract Res Clin Obstet Gynaecol 24(3): 339-352, 2010. PMID: 20097136. DOI: 10.1016/j.bpobgyn.2009.11.010

17 Provan D, Stasi R, Newland AC, Blanchette VS, Bolton-Maggs P, Bussel JB, Chong BH, Cines DB, Gernsheimer TB, Godeau B, Grainger J, Greer I, Hunt BJ, Imbach PA, Lyons G, McMillan R, Rodeghiero F, Sanz MA, Tarantino M, Watson S, Young J and Kuter DJ: International consensus report on the investigation and management of primary immune thrombocytopenia. Blood 115(2): 168-186, 2010. PMID: 19846889. DOI: 10.1182/blood2009-06-225565

18 Ranucci M, Baryshnikova E, Ranucci M, Silvetti S, Surgical and Clinical Outcome Research G: Fibrinogen levels compensation of thrombocytopenia-induced bleeding following cardiac surgery. Int J Cardiol 249: 96-100, 2017. PMID: 28986056. DOI: 10.1016/j.ijcard.2017.09.157

19 Engstrom G, Hedblad B, Eriksson KF, Janzon L and Lindgarde F: Complement c3 is a risk factor for the development of diabetes: A population-based cohort study. Diabetes 54(2): 570575, 2005. PMID: 15677517. DOI: 10.2337/diabetes.54.2.570

20 Peake PW, Kriketos AD, Campbell LV and Charlesworth JA: Response of the alternative complement pathway to an oral fat 
load in first-degree relatives of subjects with type II diabetes. Int J Obes (Lond) 29(4): 429-435, 2005. PMID: 15111984. DOI: 10.1038/sj.ijo.0802644

21 Figueredo A, Ibarra JL, Bagazgoitia J, Rodriguez A, Molino AM, Fernandez-Cruz A and Patino R: Plasma c3d levels and ischemic heart disease in type II diabetes. Diabetes Care 16(2): 445-449, 1993. PMID: 8432215. DOI: 10.2337/diacare 16.2.445

22 Moreno-Navarrete JM, Martinez-Barricarte R, Catalan V, Sabater M, Gomez-Ambrosi J, Ortega FJ, Ricart W, Bluher M, Fruhbeck G, Rodriguez de Cordoba S and Fernandez-Real JM: Complement factor $\mathrm{h}$ is expressed in adipose tissue in association with insulin resistance. Diabetes 59(1): 200-209, 2010. PMID: 19833879. DOI: 10.2337/db09-0700

23 Lenney JF, Peppers SC, Kucera CM and Sjaastad O: Homocarnosinosis: Lack of serum carnosinase is the defect probably responsible for elevated brain and csf homocarnosine Clin Chim Acta 132(2): 157-165, 1983. PMID: 6616870. DOI: 10.1016/0009-8981(83)90243-7

24 Thijssen E, van Caam A and van der Kraan PM: Obesity and osteoarthritis, more than just wear and tear: Pivotal roles for inflamed adipose tissue and dyslipidaemia in obesity-induced osteoarthritis. Rheumatology (Oxford) 54(4): 588-600, 2015. PMID: 25504962. DOI: 10.1093/rheumatology/keu464

25 Sauerhofer S, Yuan G, Braun GS, Deinzer M, Neumaier M, Gretz N, Floege J, Kriz W, van der Woude F and Moeller MJ: L-carnosine, a substrate of carnosinase-1, influences glucose metabolism. Diabetes 56(10): 2425-2432, 2007. PMID: 17601992. DOI: $10.2337 / \mathrm{db} 07-0177$

26 Janssen B, Hohenadel D, Brinkkoetter P, Peters V, Rind N, Fischer C, Rychlik I, Cerna M, Romzova M, de Heer E, Baelde H, Bakker SJ, Zirie M, Rondeau E, Mathieson P, Saleem MA, Meyer J, Koppel H, Sauerhoefer S, Bartram CR, Nawroth P, Hammes HP, Yard BA, Zschocke J and van der Woude FJ: Carnosine as a protective factor in diabetic nephropathy: Association with a leucine repeat of the carnosinase gene cndp1. Diabetes 54(8): 2320-2327, 2005. PMID: 16046297. DOI: 10.2337/diabetes.54.8.2320

27 Herrera-Marcos LV, Lou-Bonafonte JM, Martinez-Gracia MV, Arnal C, Navarro MA and Osada J: Prenylcysteine oxidase 1, a pro-oxidant enzyme of low density lipoproteins. Front Biosci (Landmark Ed) 23: 1020-1037, 2018. PMID: 28930587. DOI: $10.2741 / 4631$

28 Qiu X, Wei X, Guan H, Su H, Gong J, Fang K, Zou X, Dong H, $\mathrm{Xu} \mathrm{L}$ and $\mathrm{Lu} \mathrm{F}$ : Comparative proteomic analysis of two differently extracted coptis chinensis in the treatment of type 2 diabetic rats. Evid Based Complement Alternat Med 2018: 3248521, 2018. PMID: 30302116. DOI: 10.1155/2018/3248521

29 Oliva K, Barker G, Rice GE, Bailey MJ and Lappas M: 2d-dige to identify proteins associated with gestational diabetes in omental adipose tissue. J Endocrinol 218(2): 165-178, 2013. PMID: 23709000. DOI: 10.1530/JOE-13-0010

30 Nikitovic D, Katonis P, Tsatsakis A, Karamanos NK and Tzanakakis GN: Lumican, a small leucine-rich prote.oglycan. IUBMB Life 60(12): 818-823, 2008. PMID: 18949819. DOI: 10.1002/iub.131
31 Chakravarti S: Functions of lumican and fibromodulin: Lessons from knockout mice. Glycoconj J 19(4-5): 287-293, 2002. PMID: 12975607. DOI: 10.1023/A:1025348417078

32 Fan W, Liu T, Chen W, Hammad S, Longerich T, Hausser I, Fu Y, Li N, He Y, Liu C, Zhang Y, Lian Q, Zhao X, Yan C, Li L, Yi C, Ling Z, Ma L, Zhao X, Xu H, Wang P, Cong M, You H, Liu Z, Wang Y, Chen J, Li D, Hui L, Dooley S, Hou J, Jia J and Sun B: Ecm1 prevents activation of transforming growth factor beta, hepatic stellate cells, and fibrogenesis in mice. Gastroenterology 157(5): 1352-1367 e1313, 2019. PMID: 31362006. DOI: 10.1053/j.gastro.2019.07.036

33 Lee KM, Nam K, Oh S, Lim J, Kim YP, Lee JW, Yu JH, Ahn SH, Kim SB, Noh DY, Lee T and Shin I: Correction to: Ecm1 regulates cell proliferation and trastuzumab resistance through activation of egf-signaling. Breast Cancer Res 21(1): 45, 2019. PMID: 30914052. DOI: 10.1186/s13058-019-1106-3

34 Yamashita Y, Nakada S, Yoshihara T, Nara T, Furuya N, Miida T, Hattori $\mathrm{N}$ and Arikawa-Hirasawa E: Perlecan, a heparan sulfate proteoglycan, regulates systemic metabolism with dynamic changes in adipose tissue and skeletal muscle. Sci Rep 8(1): 7766, 2018. PMID: 29773865. DOI: 10.1038/s41598-01825635- $\mathrm{x}$

35 Stenina-Adognravi $\mathrm{O}$ and Plow EF: Thrombospondin-4 in tissue remodeling. Matrix Biol 75-76: 300-313, 2019. PMID: 29138119. DOI: $10.1016 /$ j.matbio.2017.11.006

36 Muppala S, Frolova E, Xiao R, Krukovets I, Yoon S, Hoppe G, Vasanji A, Plow E and Stenina-Adognravi O: Proangiogenic properties of thrombospondin-4. Arterioscler Thromb Vasc Biol 35(9): 1975-1986, 2015. PMID: 26139464. DOI: 10.1161/ ATVBAHA.115.305912

37 Muppala S, Xiao R, Krukovets I, Verbovetsky D, Yendamuri R, Habib N, Raman P, Plow E and Stenina-Adognravi O: Thrombospondin-4 mediates tgf-beta-induced angiogenesis. Oncogene 36(36): 5189-5198, 2017. PMID: 28481870. DOI: 10.1038/onc.2017.140

38 Zierfuss B, Hobaus C, Herz CT, Pesau G, Koppensteiner R and Schernthaner GH: Thrombospondin-4 increases with the severity of peripheral arterial disease and is associated with diabetes. Heart Vessels, 2019. PMID: 31227875. DOI: 10.1007/s00380019-01453-7

39 Correa PJ, Venegas P, Palmeiro Y, Albers D, Rice G, Roa J, Cortez J, Monckeberg M, Schepeler M, Osorio E and Illanes SE: First trimester prediction of gestational diabetes mellitus using plasma biomarkers: A case-control study. J Perinat Med 47(2): 161-168, 2019. PMID: 30205647. DOI: 10.1515/jpm-2018-0120

40 Fatema N, Deeba F, Akter S, Sultana N, Nasrin B, Ali L and Begum SA: Crp (c-reactive protein) in early pregnancy predictor for development of gdm. Mymensingh Med J 25(2): 271-276, 2016. PMID: 27277359.
Received December 13, 2019

Revised December 27, 2019

Accepted December 27, 2019 